\title{
Estimación de la deformación superficial de las laderas del volcán Turrialba (Costa Rica) después de una erupción mediante interferometría diferencial de imágenes radar de apertura sintética (SAR) Sentinel-1A
}

\author{
Estimation of the surface deformation of the slopes of the \\ Turrialba volcano (Costa Rica) after an eruption using \\ Sentinel-1A differential synthetic aperture radar interferometry \\ (SAR)
}

\author{
Rubén Martínez Barbáchano ${ }^{1}$ \\ Universidad de Costa Rica, Costa Rica
}

\begin{abstract}
Resumen
La interferometría diferencial con radar de apertura sintética (SAR) es una técnica conocida en teledetección por sus aplicaciones en la generación de modelos digitales del terreno y el monitoreo de deformaciones de la corteza terrestre. Esta técnica se basa en la fase, un parámetro presente en las imágenes SAR y sensible a la topografía del terreno. Las diferencias de fase se relacionan con cambios de elevación ocurridos entre dos barridos del satélite sobre un mismo espacio. Para la obtención de información topográfica se requiere un modelo digital del terreno y la diferencia de fase permite realizar estimaciones de desplazamiento vertical del terreno con precisiones superiores a 1 centímetro. El presente trabajo permitió estimar la deformación superficial experimentada en la ladera oeste del cráter del volcán Turrialba tras la erupción del 1 de agosto de 2020. Para ello se utilizaron varias imágenes captadas por el sensor Sentinel 1A, de la Agencia Espacial Europea.
\end{abstract}

Palabras clave: Teledetección, radar, SAR, interferometría, vulcanismo

1 Geógrafo especializado en Teledetección y Sistemas de Información Geográfica. Escuela de Geografía, Universidad de Costa Rica, Costa Rica. Correo electrónico: rubenztm@gmail.com iD https://orcid. org/0000-0002-9176-3112 


\begin{abstract}
Differential synthetic aperture radar interferometry (SAR) is a technique known in remote sensing for its applications in the generation of digital terrain models and the monitoring of deformations of the earth's crust. This technique is based on the phase, a parameter present in SAR images and sensitive to terrain topography. Phase differences are related to elevation changes that occur between two satellite scans over the same space. To obtain topographic information, a digital terrain model is required and thus, phase differences provide information for estimating vertical terrain displacement to an accuracy greater than 1 centimeter. In the present work it was possible to estimate the surface deformation that occurred on the western slope of the crater of the Turrialba volcano after the eruption of August 1, 2020. For this purpose, several images captured by the Sentinel-1A sensor of the European Space Agency were used.
\end{abstract}

Keywords: Remote sensing; Radar; SAR; Interferometry; Volcanism.

\title{
Introducción
}

La Agencia Espacial Europea (European Space Agency-ESA) tiene en marcha el programa Copérnico, un ambicioso proyecto espacial de observación de la tierra, que desde 2011 proporciona información de la superficie terrestre y de la atmósfera de forma sistemática, actualizada y de fácil acceso, con el objetivo de comprender y mitigar los efectos del cambio climático, a través de los datos procedentes de más de 30 satélites. Entre dichos satélites, cabe mencionar las cinco familias de misiones Sentinel, comenzando por las imágenes radar de Sentinel-1, que desde 2014 nos proporciona observaciones de la superficie de la Tierra en cualquier condición meteorológica, de día o de noche. La teledetección mediante radar de apertura sintética (SAR) ha experimentado un renovado impulso en los últimos años, con nuevos sensores y plataformas que han permitido la obtención de magnitudes o variables geofísicas de enorme valor geográfico. Desde el punto de vista del espectro electromagnético, los sistemas RADAR trabajan en el rango de las microondas como sensores activos, debido a que la emitancia de los objetos es difícilmente perceptible en esa banda (Chuvieco, 2010).

Entre las técnicas recientes más utilizadas con tecnología radar destaca la interferometría. Esta técnica ha permitido, tanto a escala regional como local, medir deformaciones centimétricas, e incluso milimétricas, de la superficie topográfica bajo ciertas condiciones. En este sentido destacan las aplicaciones en desplazamientos sísmicos relacionados con fallas activas (Massonet et al., 1993), movimientos de masas glaciares (Goldstein, Zebker \& Werner, 1988) o deformaciones relacionadas con la actividad 
volcánica e intrusiones de magma (Sigmudsson, Durand \& Massonnet, 1999). También cabe mencionar estudios de subsidencia kárstica (Guerrero, 2008), minera (Herrera et al., 2007), urbana (Cascini et al. 2006), o la producida por extracción de aguas subterráneas (Amelung, Galloway, Bell, Zebker \& Laczniak, 1999).

\section{Marco conceptual}

Los sistemas RADAR basan su funcionamiento en una señal electromagnética emitida a través de una antena, en sentido lateral a la plataforma. La señal se dispersa y choca con la superficie u objeto al que va dirigido y la antena radar capta una parte de esas ondas reflejadas. El sistema, por tanto, se considera teledetección activa, al utilizar la información de la señal emitida y la información de las señales reflejadas para construir una imagen de la zona observada.

Desde el punto de vista de la longitud de onda, estos sistemas trabajan en una banda comprendida entre los $0,1 \mathrm{~cm}$ y $1 \mathrm{~m}$. Aunque la observación de la superficie terrestre en esta longitud de onda entraña dificultades complejas y diferentes a las del espectro óptico, su principal ventaja reside en que es prácticamente transparente a la cobertura nubosa y las influencias atmosféricas, debido a que se basa en longitudes de onda mayores a $0,1 \mathrm{~mm}$, que es el tamaño habitual de las gotas de agua. El hecho de que la teledetección con microondas se base en el uso de sensores activos se debe a la escasa emisividad espectral que se da en esta región del espectro, como demuestra la de Planck calculada por Rayleigh-Jeans:

$$
\mathrm{M}_{\mathrm{n} \lambda}=2 \pi \mathrm{k} T / \lambda^{4}
$$

Donde Mn $\lambda$ es la emitancia espectral, $T$ es la temperatura en grados Kelvin y $\lambda$ es la longitud de onda. La anterior expresión demuestra que la emitancia de los objetos es difícilmente detectable en la región de las microondas, razón por la cual la teledetección activa mediante radar es la más extendida en usos y aplicaciones.

Los píxeles que conforman una imagen radar representan el coeficiente de retrodispersión de la superficie del terreno, con valores directamente proporcionales a la intensidad de la señal de retorno. A diferencia de los sensores pasivos que trabajan en el espectro óptico, la principal virtud 
o ventaja que ofrece la tecnología radar es que puede trabajar en cualquier condición atmosférica (Chuvieco 2010).

Un sensor radar puede configurarse de muy diversas formas, obteniendo así señales de retorno igualmente diversas. De hecho, resulta habitual encontrar sensores radar con distintas bandas de observación, diferentes alturas, diferentes ángulos de observación, diferentes polarizaciones y antenas de tamaño diverso.

La ecuación fundamental del radar es la siguiente:

$$
P_{r}=P_{t} G^{2} \lambda^{2} \sigma /(4 \pi)^{3} r^{4}
$$

Siendo $\operatorname{Pr}$ la potencia de la señal retrodispersada, $P t$ la potencia emitida por el sensor, $G$ el factor de ganancia de la antena, $\sigma$ la sección eficaz de retrodispersión y $r$ la distancia entre el sensor y la cubierta. De la expresión anterior se puede deducir que la señal de retorno es altamente dependiente de la potencia emitida y de la altura de observación (Chuvieco2010). Tal afirmación puede confirmarse a través de la siguiente ecuación:

$$
P_{r}=P_{t} A^{2} \sigma / 4 \pi \lambda^{2} r^{4}
$$

En la que $A$ representa la superficie de la antena receptora, lo que implica que, a mayor altura de observación del sensor, el tamaño de la antena debería ser mayor para obtener una buena señal. Por ello, a la hora de incorporar sensores radar a plataformas orbitales se requiere de antenas de gran tamaño, que garanticen una gran potencia o elevada ganancia efectiva. Ello plantea un reto logístico o de diseño, dado que para obtener una resolución aceptable los sensores orbitales deberían contar con enormes antenas. Pero este problema puede solventarse en parte con el diámetro de apertura de la antena, representado por $\Phi$ en la siguiente expresión:

$$
R_{s}=\lambda r / \Phi
$$

El radar de apertura sintética (SAR) permite simular distintos tamaños de antena mediante el denominado efecto Doppler, consistente en que la resolución del sensor se basa en la obtención de dos señales de retorno de un mismo punto de la superficie terrestre en dos instantes diferentes de 
la trayectoria del sensor. De este modo, la resolución no depende del tamaño real de la antena, sino de la que se obtendría de una antena de la misma longitud a la distancia existente entre esos dos puntos (Elachi, 1987).

Figura 1. Geometría de observación de una imagen SAR. El radar vuela a largo de una línea de altitud $\mathrm{H}$ y observa la superficie terrestre con un ángulo oblicuo $\theta_{l}$

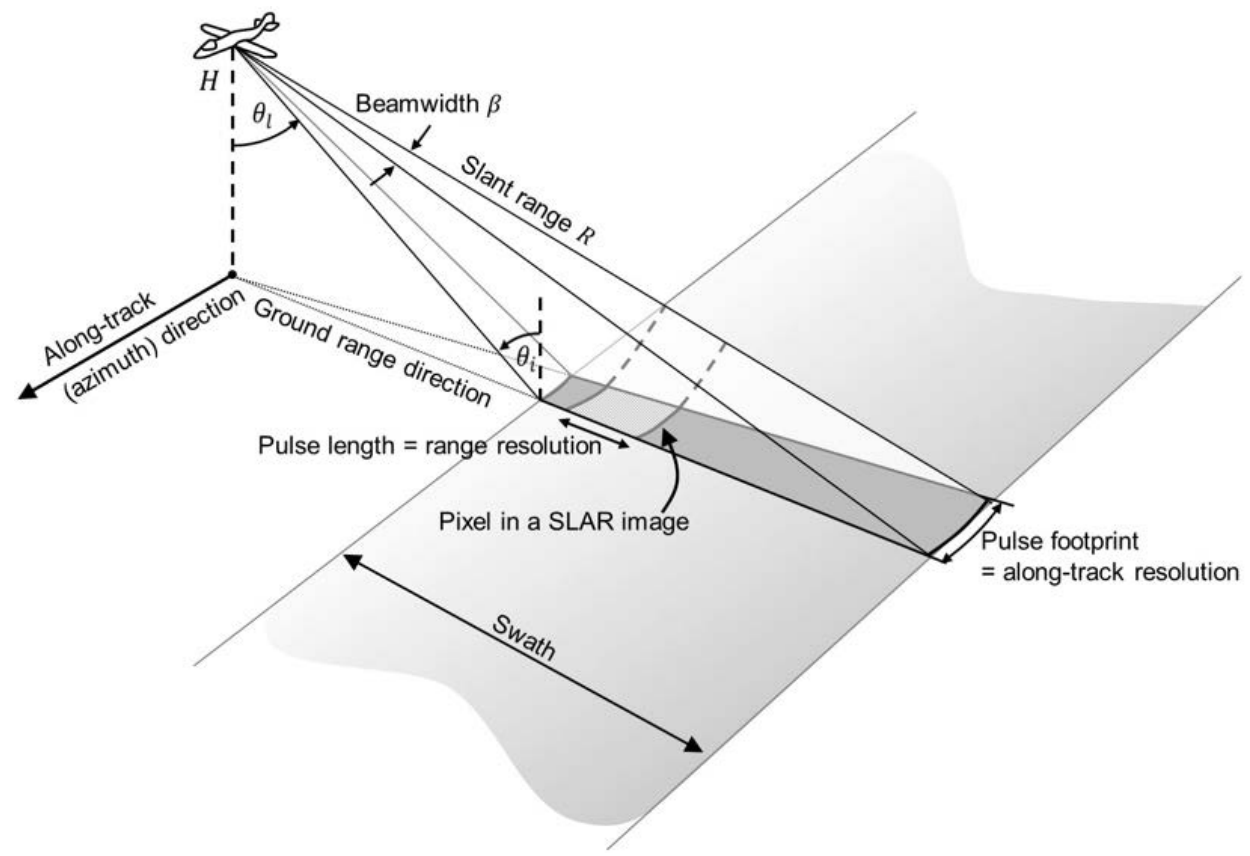

Fuente: Siqueira, 2019.

Dado que la imagen se forma con las lecturas de las señales retrodispersadas en la superficie y que son captadas y procesadas por la antena del satélite, se genera en cada punto de la imagen un número complejo:

$$
\begin{aligned}
& \mathrm{i}=\mathrm{A}^{*} \cos \varphi \\
& \mathrm{q}=\mathrm{A}^{*} \operatorname{sen} \varphi
\end{aligned}
$$

Siendo i la parte real y q la imaginaria, A la amplitud de la señal y $\boldsymbol{\varphi}$ la fase medida. 
Estos dos valores son los existentes en cada píxel de la imagen, en consecuencia, se puede extraer la información de fase e intensidad del punto:

$$
\begin{aligned}
& \mathrm{A}=\sqrt{ } i 2+q 2 \\
& \Phi=\operatorname{atan}(q / i) \\
& \mathrm{Int}=i^{2}+q^{2}
\end{aligned}
$$

La interferometría se centra en el estudio y explotación de la información proporcionada por las imágenes de fase de las imágenes complejas SAR (Hanssen, 2001). Tanto la técnica de interferometría SAR (InSAR) como la interferometría diferencial (DInSAR) se basan en la explotación contenida en uno o más interferogramas (Klees \& Massonnet, 1999). Concretamente, en obtener la posición de un punto hipotético, en función de la observación (desde un radar SAR) de dicho punto desde dos posiciones distintas. Por tanto, las imágenes SAR contienen en cada píxel información de la amplitud y la fase correspondiente a los elementos del terreno. La amplitud varía en función de las características de que la zona de estudio ejerce sobre la señal retrodispersada, mientras que la fase contiene la información de la distancia de la antena al suelo de la zona de estudio (Moreira, et al., 2013).

Un interferograma se forma con dos imágenes SAR, mediante un proceso en el cual cada píxel de la primera imagen se multiplica por el conjugado del mismo píxel de la segunda imagen, obteniéndose una nueva imagen en la que los valores que contiene son la multiplicación de los valores de la amplitud y la diferencia de fase en cada uno de los píxeles de una imagen respecto de la otra.

En la señal retrodispersada que recibe el sensor, además de la distancia al punto y las características de la superficie, influyen otros factores como el ruido inherente al sistema de medida o las condiciones atmosféricas, que influyen en la calidad del producto obtenido (Moreira et al., 2013).

Tal y como ha sido expuesto, por tanto, se puede determinar la posición de un punto mediante el cálculo de la variación de distancia que existe al observar el punto desde dos posiciones distintas del satélite, pudiendo ocurrir también que la variación del valor de distancia se deba a una variación en la posición del punto. 
Por todo ello, para poder aislar y cuantificar el valor de la diferencia de fase correspondiente al desplazamiento del punto nació la interferometría diferencial SAR conocida como DinSAR. Su rasgo más característico es que elimina los efectos topográficos incluidos en la fase del punto utilizando un modelo digital de elevaciones, generando lo que se conoce como un interferograma diferencial. Al suprimir el componente topográfico del valor de la fase quedarían otros factores que contribuyen al desplazamiento sufrido por el punto; se trata de las condiciones atmosféricas y el ruido de fase debido a la decorrelación temporal y variaciones del medio de propagación. (Moreira et al., 2013).

En cuanto a la capacidad y límites de esta técnica en la identificación de procesos volcánico-sísmicos como el que nos ocupa, existe un límite interferométrico que viene dado por la expresión:

\section{$2 L(\operatorname{sen} \varphi 1-\operatorname{sen} \varphi 2)<\lambda$}

Donde $\mathbf{L}$ es la longitud del píxel perpendicular a la trayectoria, $\boldsymbol{\varphi} \mathbf{1}$ y $\boldsymbol{\varphi} 2$ son los ángulos de incidencia (desde la vertical local) en la primera y

Figura 2. Representación de algunos procesos geológicos susceptibles de ser detectados por INSAR, en color verde

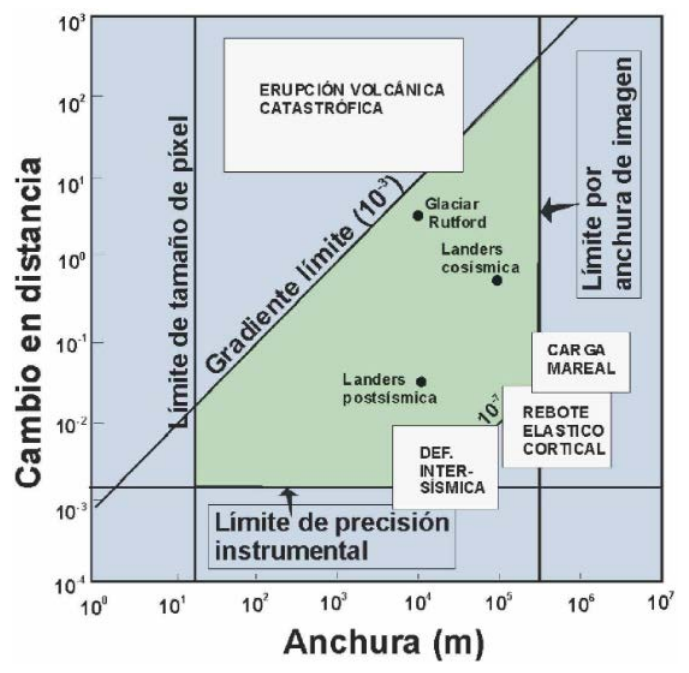

Fuente: Jiménez-Perálvarez (2013). segunda imagen comparada, $y$ $\lambda$ es la longitud de onda de la radiación emitida.

La figura 2 relaciona el cambio de distancia o deformación producida por el proceso, estimada en metros para la zona afectada. El "gradiente límite" representa el máximo gradiente de desplazamiento que puede llegar a identificarse con esta técnica (Massonnet \& Feigl, 1998).

\section{Área geográfica de estudio \\ El volcán Turrialba se lo- caliza en la provincia de Car- tago, en el cantón de Turrialba}


(distrito de Santa Cruz). Se trata de un estratovolcán complejo y activo, que forma parte del extremo oriental de la cordillera Central de Costa Rica. Con una elevación máxima de $3340 \mathrm{~m}$, es el segundo volcán más alto del país, presenta una temperatura promedio anual de $16{ }^{\circ} \mathrm{C}$ y una precipitación de $3000 \mathrm{~mm}$ a $4000 \mathrm{~mm}$ anuales. Las laderas occidentales, objeto del presente estudio, se encuentran situadas en las coordenadas $10^{\circ} 0$ ' $58^{\prime \prime}$ de latitud norte y $83^{\circ} 46^{\prime} 37^{\prime \prime}$ oeste a una altitud de 2796 metros, con pendientes que oscilan entre el $12 \%$ y el $50 \%$.

La erupción magmática más reciente que se conoce tuvo lugar entre los años 1864-1866, con un extenso período de calma relativa posterior. Sin embargo, comenzó a mostrar signos de reactivación a partir del mes de marzo del 2012, con una fuerte actividad fumarólica, gases a altas temperaturas, incandescencia, lluvia ácida y ocasionales erupciones freáticas, por lo que algunos pobladores ubicados al sur, suroeste y oeste del volcán han tenido que abandonar sus hogares (González et al., 2015). Entre mayo y junio de 2016 se produjeron al menos tres erupciones estrombolianas, alternadas con períodos de calma., siendo la más reciente la del 1 de agosto de 2020, que provocó una columna de ceniza de 500 metros de altura. Según el Boletín semanal del Programa de Vigilancia Volcánica (del 3 de agosto del 2020) del Observatorio Vulcanológico y Sismológico de Costa Rica (OVSICORI, 2020) se observaron "varias emisiones de ceniza diluidas, y al menos un pulso corto un poco más denso, con una actividad sísmica que presenta un nivel de fondo estable desde al menos enero del 2020 y una tendencia a la contracción y la subsidencia del macizo Turrialba-Irazú que continúa" (OVSICORI, 2020, p. 1).

\section{Materiales y métodos}

Para el presente trabajo se utilizaron exclusivamente recursos libres online y software de código abierto. Todo el procesamiento de imágenes que sustenta la presente metodología se realizó con el software SNAP desarrollado por la Agencia Espacial Europea (Figura 3).

Dado que la técnica DInSAR (InSAR diferencial) se basa en los cambios de elevación ocurridos entre dos barridos del satélite sobre una misma área, el primer paso consistió en escoger las imágenes inmediatamente anteriores y posteriores a la fecha de erupción del volcán, el 1 de agosto de 2020. Las imágenes Sentinel 1 están disponibles para libre descarga en 
Rubén Martínez Barbáchano

Estimación de la deformación superficial de las laderas del volcán Turrialba (Costa Rica) después de una erupción mediante interferometría diferencial de imágenes radar de apertura sintética (SAR) Sentinel-1A

Figura 3. Software SNAP en su versión 8.0.2

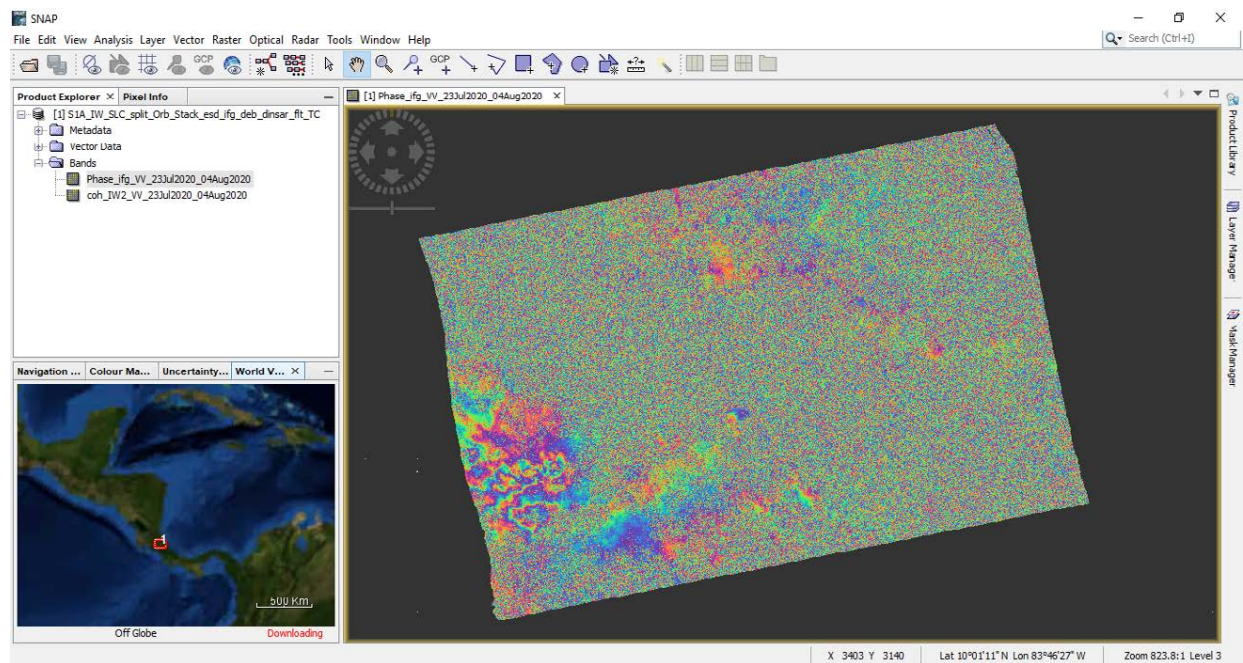

el sitio web Copernicus Open Access Hub (anteriormente conocido como Sentinels Scientific Data Hub) que proporciona acceso completo, gratuito y abierto a los productos de usuario de las misiones Sentinel-1, Sentinel-2, Sentinel-3 y Sentinel-5P.

Figura 4. Sitio web Copernicus Open Access Hub

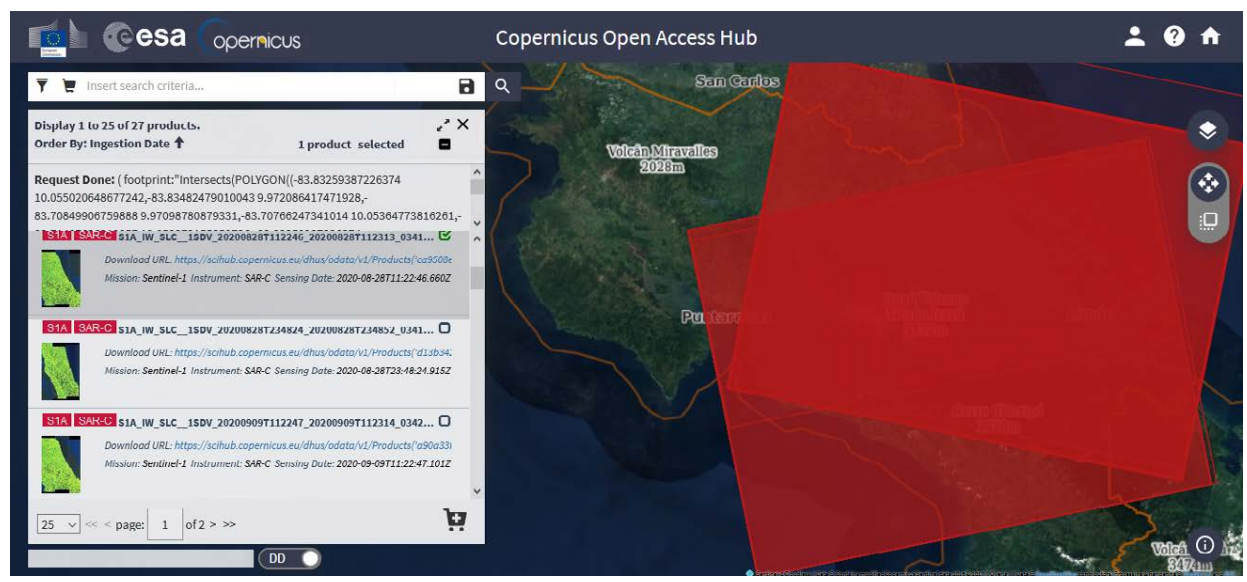

Fuente: https://scihub.copernicus.eu/dhus/\#/home 
Las imágenes utilizadas correspondían al 23 de julio y al 04 de agosto de 2020, del satélite Sentinel 1A, nivel de producto SLC (Single-Look Complex) en modo Interferometric Wide Swath (IW) que implica un barrido de 3 franjas paralelas dirigiendo la antena a tres posiciones across-track (a lo ancho del barrido) cuyo resultado es un ancho de escena de $250 \mathrm{~km}$ con una resolución de 5 × $20 \mathrm{~m}$. Las imágenes seleccionadas para el presente estudio fueron las más cercanas al evento eruptivo del volcán, por lo que se descartaron otras imágenes Sentinel 1 de pasada ascendente, más alejadas de la fecha del evento (25 días) y, por tanto, más propensas a reflejar en la rampa de fase eventos o factores ajenos a la erupción del 1 de agosto de 2020.

El flujo de procesamiento de las imágenes radar en interferometría divide en varias fases, tal y como muestra la Tabla 1.

Tabla 1. Elaboración propia a partir de la guía para usuarios de Sentinel 1A de la Agencia Espacial Europea

\begin{tabular}{|l|l|}
\hline \multicolumn{1}{|c|}{ Tipo de procesamiento } & \multicolumn{1}{c|}{ Herramientas de software } \\
\hline 1. Corregistro de imágenes. & 1.1. Back Geocoding. \\
\cline { 2 - 3 } & 1.2. Corrección orbital. \\
\hline $\begin{array}{l}\text { 2. Creación del interferograma y estimación de } \\
\text { la coherencia. }\end{array}$ & 2. Interferogram Formation \\
\hline 3. Eliminación de bandeado. & 4. Herramienta TOPS Deburst. \\
\hline 5. Reducción del ruido de la imagen. & 5. Filtro Goldstein. \\
\hline 6. Desenrollado de franjas de la imagen de fase. & 6. Plugin SNAPHU Unwrapping \\
\hline 7. Conversión de la fase a desplazamiento. & 7. Herramienta Phase to Displacement \\
\hline 8. Corrección geométrica. & 8. Range Doppler Terrain Correction \\
\hline \multirow{2}{*}{ 9. Generación de productos finales. } & 9.1. Aplicación de máscara de coherencia. \\
\cline { 2 - 2 } & 9.2. Exportar a formatos cartográficos. \\
\hline
\end{tabular}

Fuente: https://sentinel.esa.int/web/sentinel/user-guides/sentinel-1-sar/product-types-processinglevels/level-1

Como paso previo al procesamiento, se realizó un subset consistente en seleccionar únicamente las franjas de barrido de ambas imágenes que abarcaban el área de estudio, mediante la herramienta S-1 TOPS Split, tal y como muestra la Figura 5.

Una vez realizado el subset de franjas de barrido, el siguiente paso fue el corregistro de imágenes obtenidas en el paso anterior, pero antes es necesario aplicar en las imágenes obtenidas anteriormente los archivos con la información detallada de las órbitas, mediante la herramienta de SNAP “Apply Orbit File" tal y como muestra la Figura 6. 
Rubén Martínez Barbáchano

Estimación de la deformación superficial de las laderas del volcán Turrialba (Costa Rica) después de una erupción mediante interferometría diferencial de imágenes radar de apertura sintética (SAR) Sentinel-1A

Figura 5. Creación de un subset con las franjas de barrido del área de estudio

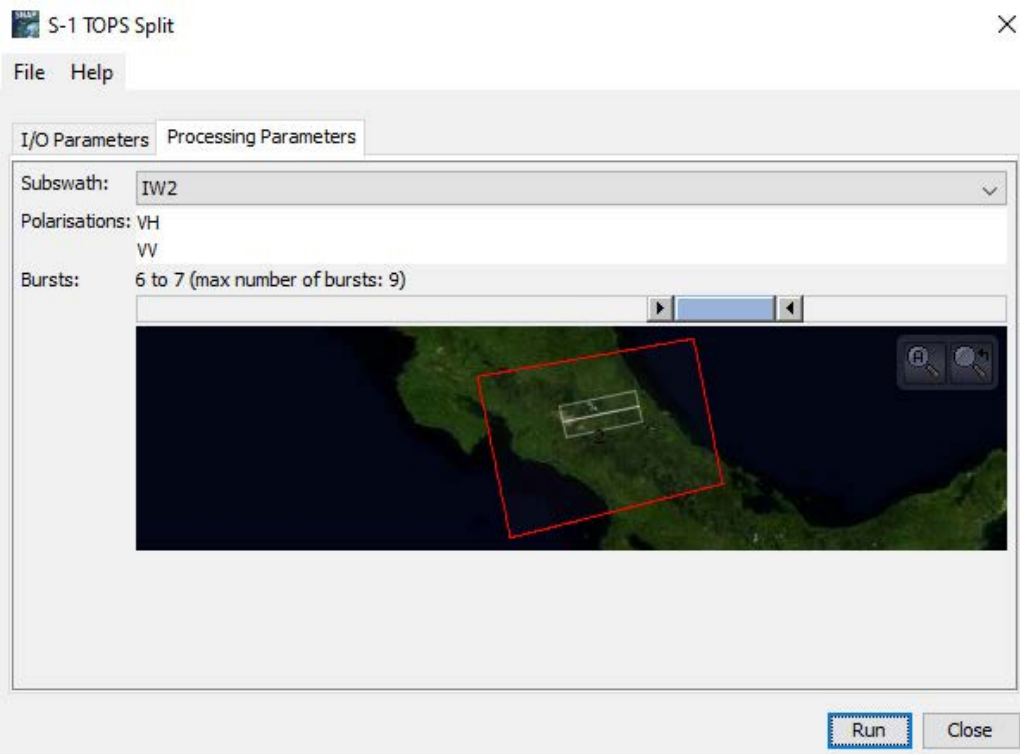

\section{Figura 6. Aplicación de la información de órbita}

C. Apply Orbit File

File Help

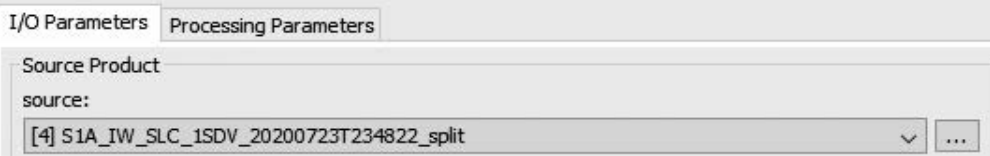

$\square$ Open in SNAP 
Una vez aplicada la información de la trayectoria orbital en las imágenes, se procede a aplicar el corregistro. Se trata de un proceso consistente en posicionar los píxeles de una imagen (la del 23 de julio) con los mismos píxeles correspondientes a otra imagen (la del 04 de agosto) para poder generar correctamente un interferograma. Para ello se selecciona una imagen (normalmente la más antigua) como imagen de referencia y la otra imagen (la más reciente) se selecciona como subordinada. La herramienta que el software SNAP proporciona para ello se denomina S-1 TOPS Corregistration.

Figura 7. Corregistro de la imagen de referencia y de la imagen subordinada

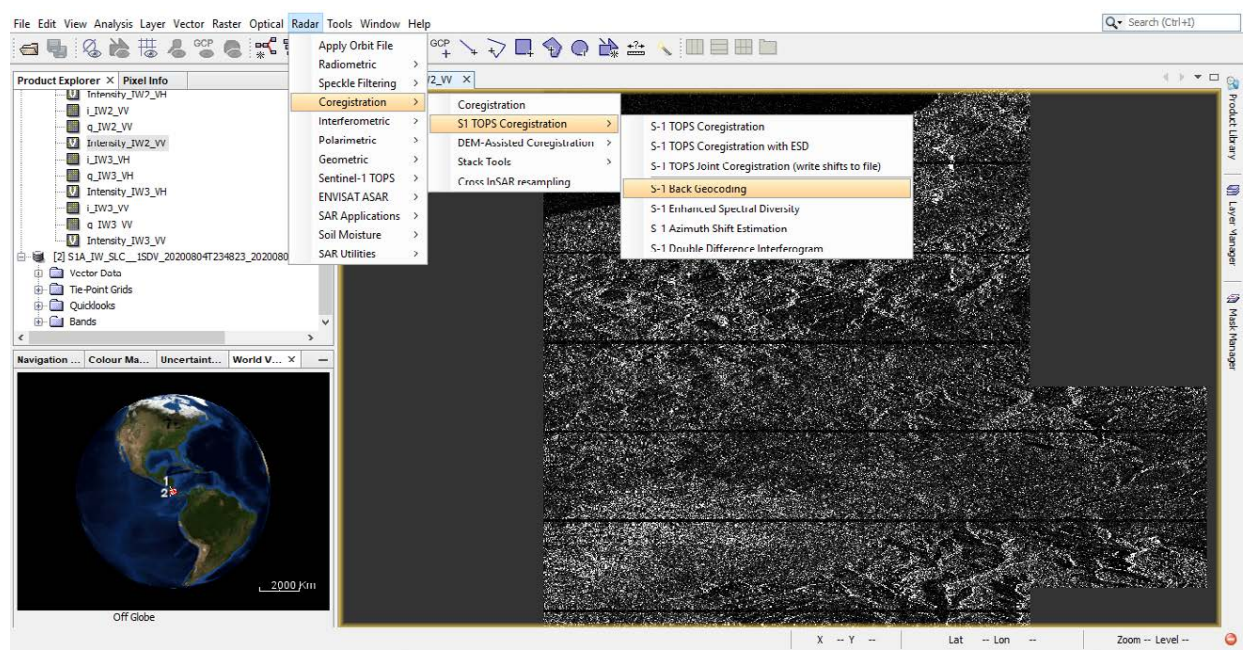

Para que este proceso se realice con éxito se requiere la información precisa de las órbitas del satélite y un modelo digital de elevación del área abarcada. SNAP permite descargar automáticamente de internet tanto las órbitas (vistas en el paso anterior) como la información topográfica (modelo digital de elevación). Para el presente trabajo se utilizó el modelo digital SRTM 1 sec HGT, un producto de la NASA (Shuttle Radar Topography Mission) generado a partir de datos radar SAR de banda $\mathrm{C}$ y X con una resolución de 3 arcosegundos, es decir, 30 metros. El método de remuestreo para alinear los puntos de la imagen subordinada con los de la imagen de referencia fue el de interpolación bilineal, recomendada para conjuntos de datos continuos sin límites definidos. Cabe mencionar por último que 
la presente técnica y el software solo permiten corregistrar una subfranja (IW2) con una única polarización (vertical-vertical).

El siguiente paso que se realizó fue la formación del interferograma. El interferograma (Figura 8) se genera al multiplicar la imagen de referencia con la imagen subordinada, lo que produce tres nuevas imágenes complejas: de amplitud, de fase y de coherencia interferométrica.

Figura 8. Interferograma generado a partir de las imágenes de 23 de julio y 04 de agosto de 2020 en el cráter del volcán Turrialba (Costa Rica)

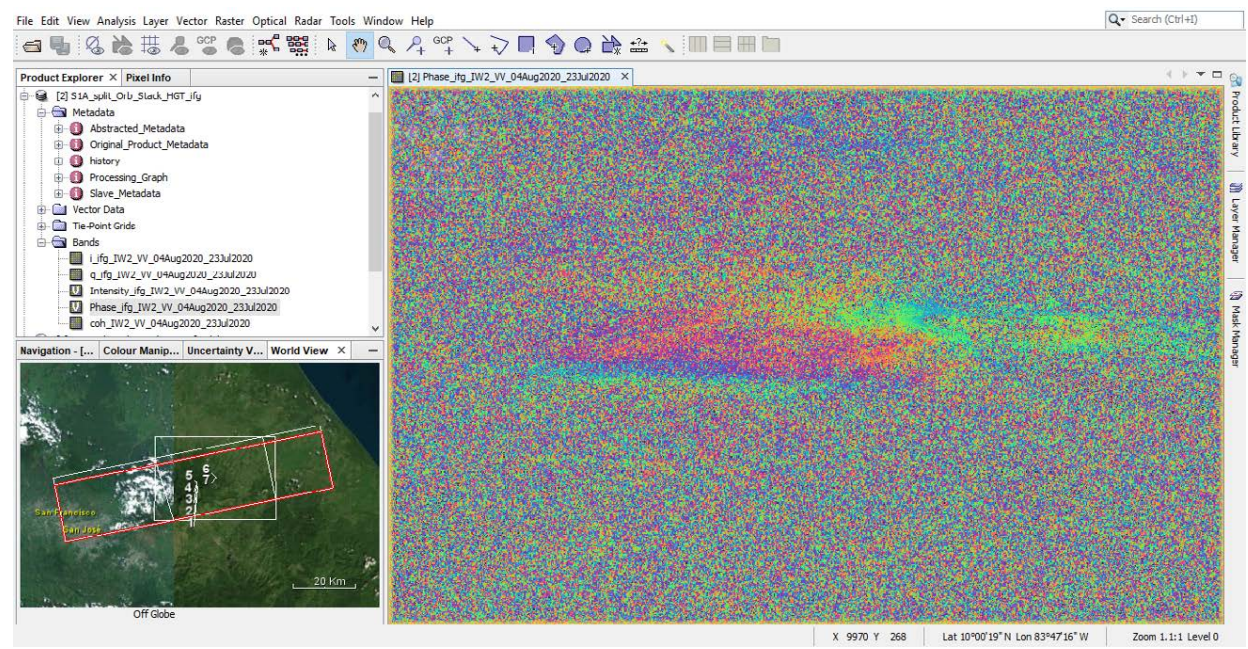

Una vez generados, los interferogramas necesitan un pre-procesado que corresponde fundamentalmente con la eliminación del ruido (speckle) $\mathrm{y}$ un posterior procesamiento de la imagen mediante un protocolo previamente establecido (Richards, Scheer \& Holm, 2010). Concretamente, y debido a la forma de adquisición de los datos Sentinel 1, las imágenes pueden presentar subfranjas dentro de la cuales se producen solapes. Para ello, el software SNAP proporciona la herramienta "S1 TOP Deburst", que une las distintas ráfagas teniendo en cuenta el solape, por lo que desaparecen las líneas intermedias, quedando una imagen continua. 
Figura 9. Imagen de intensidad de la zona de estudio con subfranja sin información, previa aplicación de la herramienta de SNAP "S1 TOP Deburst"

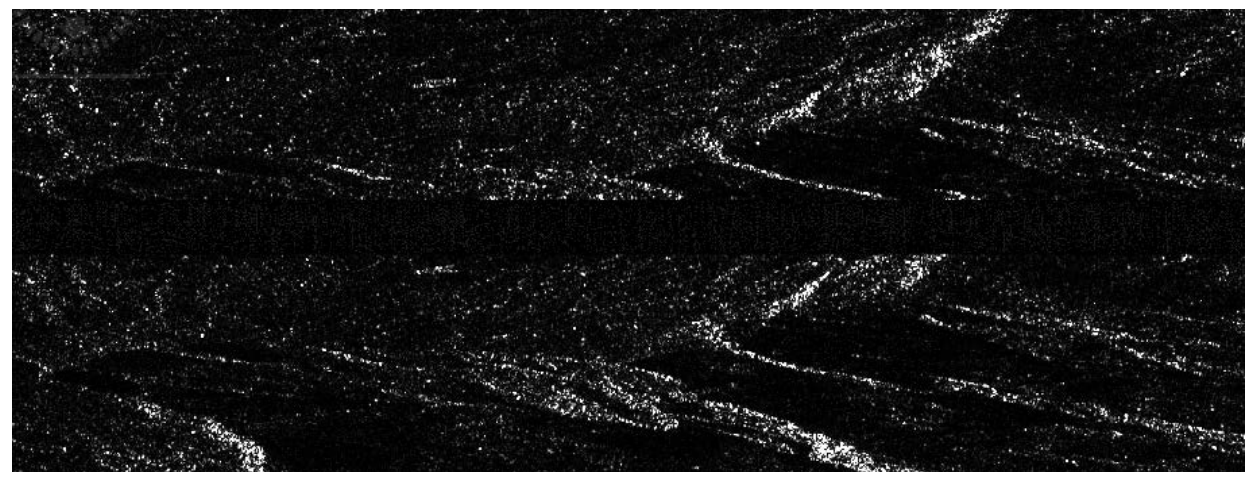

El paso siguiente consistió en la creación del interferograma diferencial, eliminando el efecto de la topografía de la zona sirviéndose de un modelo digital de elevaciones (se utilizó siempre el modelo digital SRTM 1 HGT de 3 arcosegundos). La herramienta que lo permite en SNAP es "Topographic Phase Removal".

Figura 10. Herramienta "Topographic Phase Removal” aplicada sobre el sector del volcán Turrialba

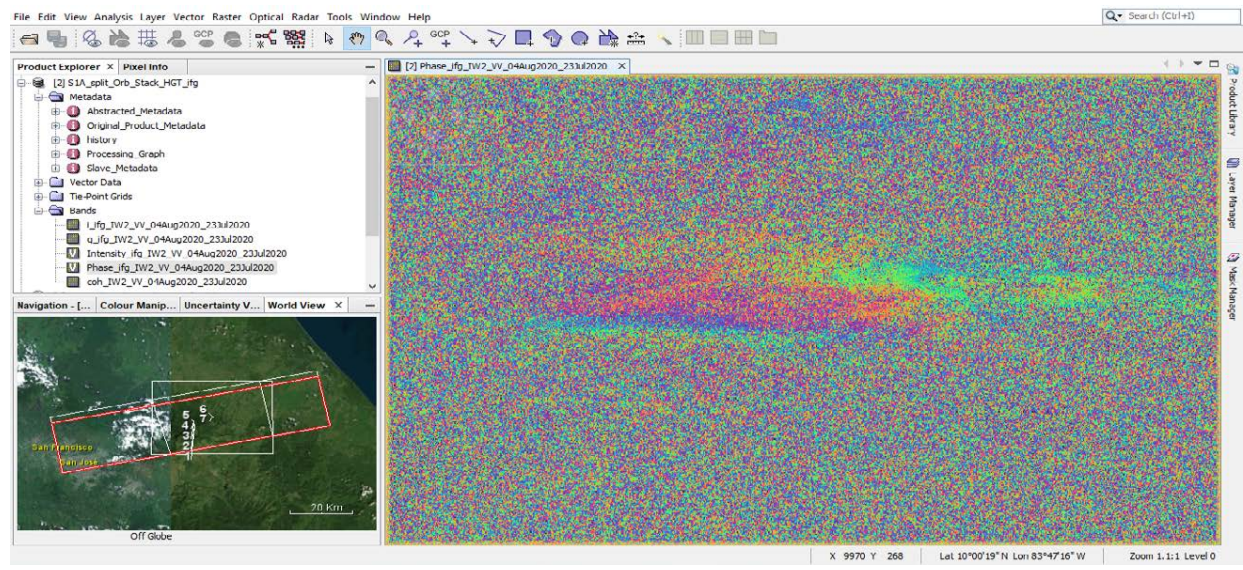

Algunos de los problemas asociados a la generación de interferogramas de fase es el ruido por problemas de decorrelación geométrica, retrodispersión y otros errores de procesamiento (Braun \& Veci, 2015). Y 
aunque la información de fase en áreas decorreladas no puede ser restituida en tales casos, la calidad de las franjas existentes en el interferograma puede ser incrementada aplicando filtros como el de Goldstein, basado en la función transformada rápida de Fourier (FFT), un algoritmo que reduce el tiempo de cálculo de $n^{2}$ pasos a $n \cdot \log _{2}(n)$ con resultados óptimos en la mayor parte de la imagen tratada, y donde no es así, el usuario es advertido por los cortes (o cambios de color) de las franjas (Goldstein et al., 1988).

De la Figura 11 podía deducirse fácilmente que el ruido de las imágenes interferométricas diferenciales dificulta su interpretación y entre los filtros disponibles en el software SNAP, destaca el citado filtro de fase de Goldstein.

Figura 11. Interferograma del volcán Turrialba antes (parte superior) y después (parte inferior) de la aplicación del filtro Goldstein

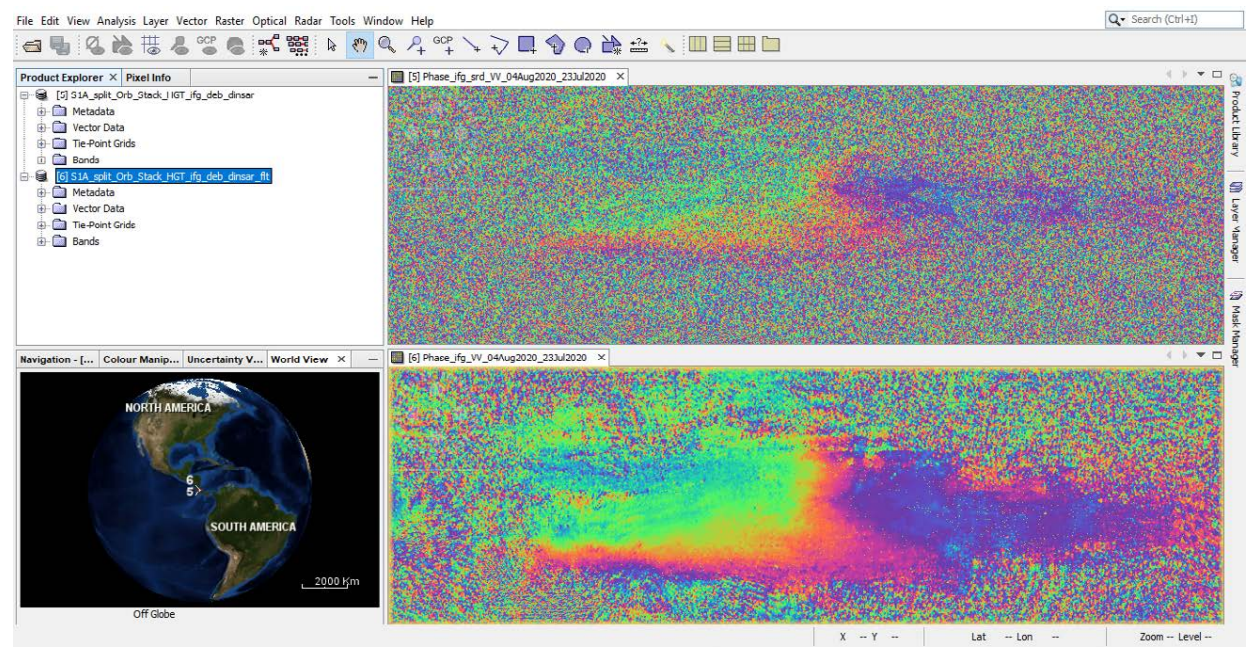

El interferograma presenta, no obstante, algunos inconvenientes aún. El principal es que para poder establecer una correlación entre la fase interferométrica y la altura topográfica hay que desenvolver el archivo correspondiente a la fase. La razón es que los valores de fase están ceñidos a un intervalo de $2 \pi$ y al desenvolver la imagen se integran la diferencia de fase entre pixeles vecinos. Por tanto, la imagen desenvuelta puede ser interpretada como la altura/desplazamiento entre pixeles de dos imágenes (Braun \& Veci, 2015). 
Figura 12. Imagen de fase sin desenvolver (izquierda) y desenvuelta (derecha) en relación con la gradación de cambios en la altura (Braun \& Veci, 2015)
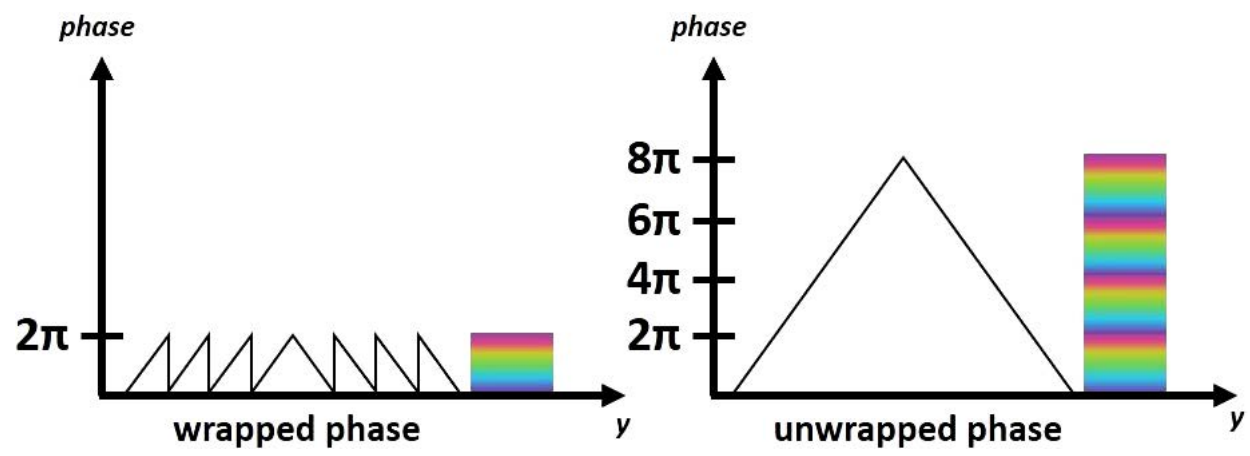

La herramienta que permitió realizar este proceso en el software de SNAP es un plugin denominado "SNAPHU Phase Unwrapping".

Figura 13. Imagen de fase desenvuelta (en radianes) con la herramienta "SNAPHU Phase Unwrapping" de SNAP

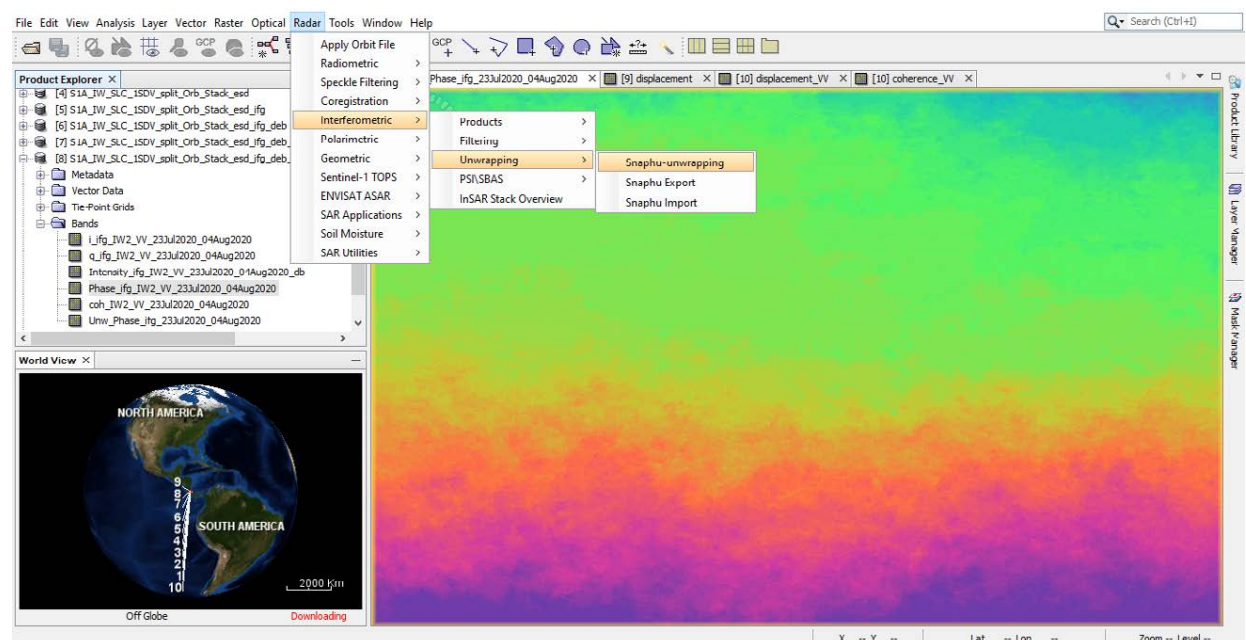

Una vez obtenido el raster de fase desenvuelto, para poder establecer relaciones paramétricas hay que convertir las unidades de la imagen de radianes en desplazamientos cuantificables. La herramienta de SNAP que permite realizar este proceso es "Phase to Displacement" y el producto 
Estimación de la deformación superficial de las laderas del volcán Turrialba (Costa Rica) después de una erupción mediante interferometría diferencial de imágenes radar de apertura sintética (SAR) Sentinel-1A

generado es una capa de tipo raster en la que cada píxel representa los valores de desplazamiento (Figura 14).

Figura 14. Histogramas de las imágenes de fase desenvuelta (izquierda) y de desplazamiento (derecha)
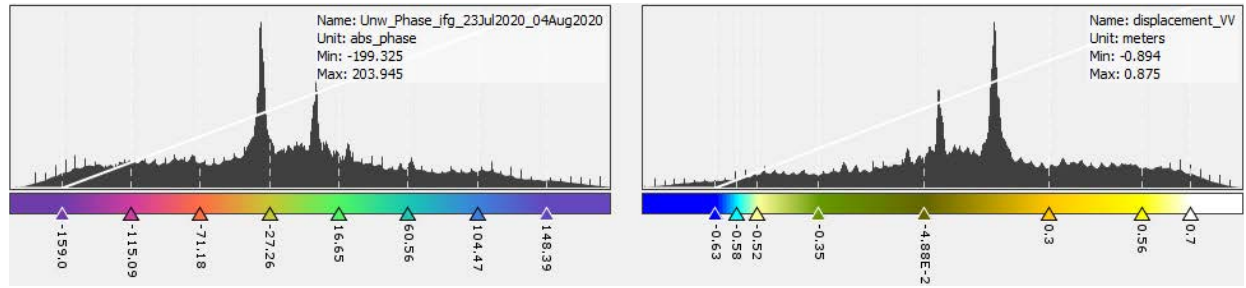

La herramienta de SNAP "Profile Plot", mediante el trazado de un transecto sobre el raster de desplazamiento, permitió confirmar que la deformación se produjo en sentido vertical ascendente y de una forma creciente hacia la base del cráter del volcán Turrialba, tal y como se muestra en la Figura 15.

Figura 15. Perfil de desplazamiento de un transecto del volcán Turrialba, mediante la herramienta de SNAP "Profile Plot"

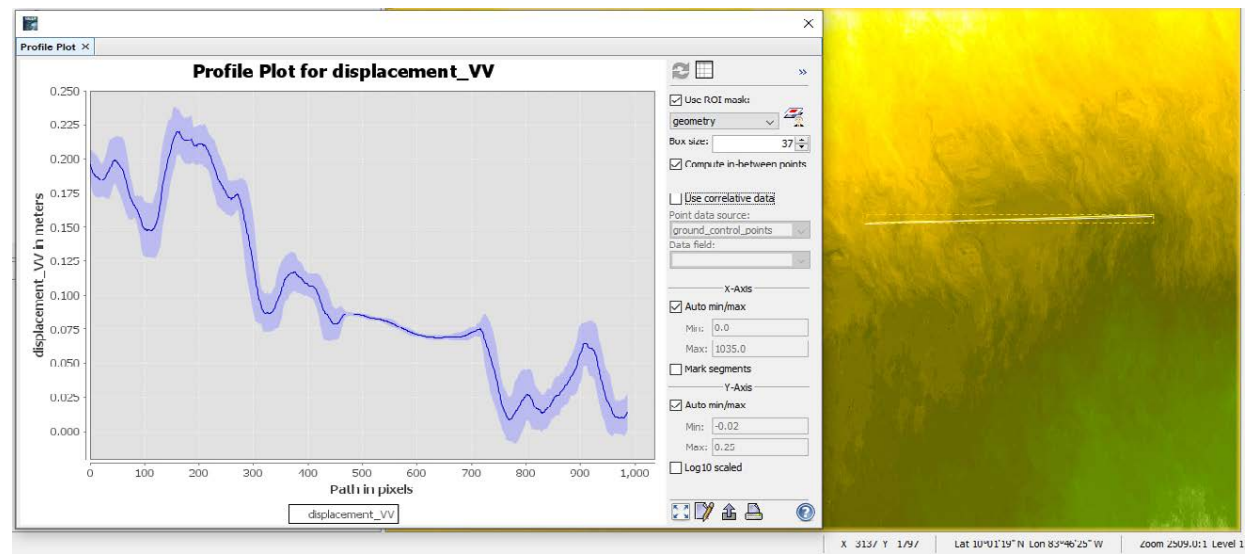

El último paso en el procesamiento interferométrico sería la corrección geométrica. La herramienta de SNAP "Range Doppler Terrain Correction" permite crear un interferograma diferencial mediante la sustracción de la topografía de la zona (como "foreshortening" o sombras 
topográficas) utilizando un modelo digital de elevaciones, en este caso el SRTM 1 HGT de 3 arcosegundos utilizado anteriormente.

Figura 16. Distorsiones geométricas en imágenes radar (Braun, 2019)

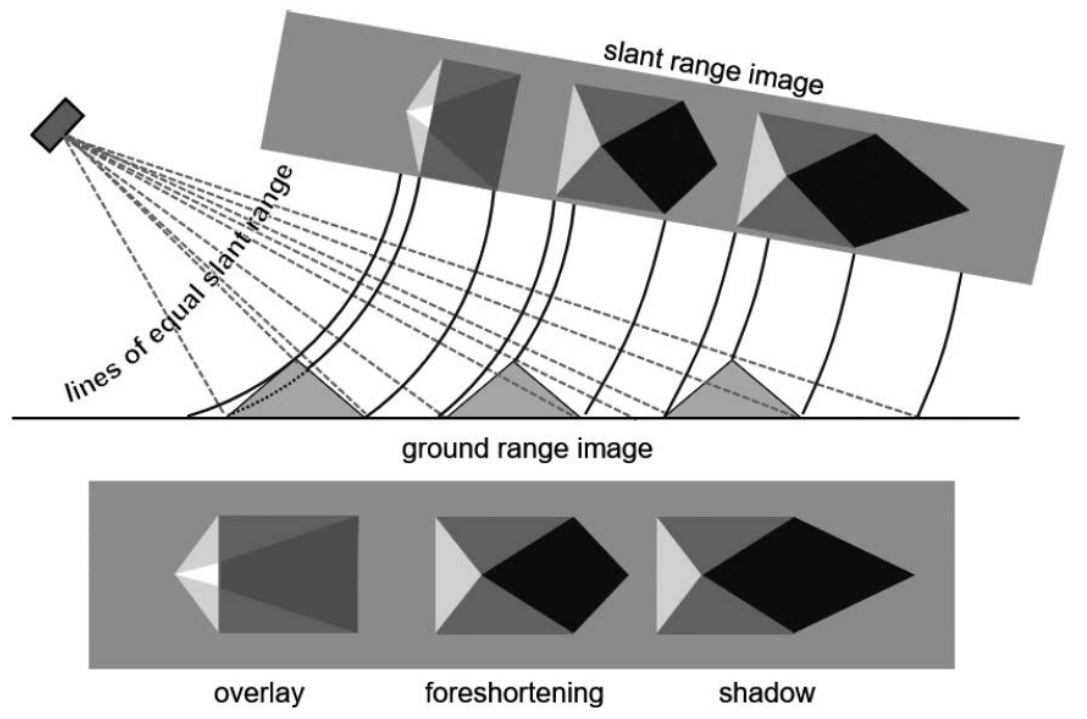

El resultado es un producto cartográfico proyectado y georreferenciado, como el que muestra la Figura 17.

Figura 17. Imágenes de coherencia (parte superior) y de desplazamiento (parte inferior) del volcán Turrialba

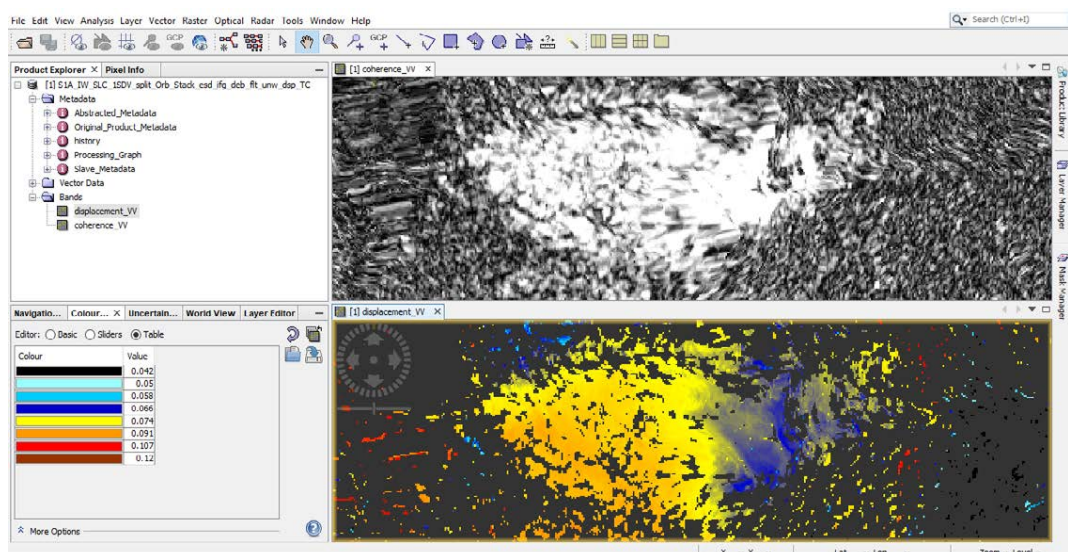


Estimación de la deformación superficial de las laderas del volcán Turrialba (Costa Rica) después de una erupción mediante interferometría diferencial de imágenes radar de apertura sintética (SAR) Sentinel-1A

Finalmente, se exportaron el raster de desplazamiento y la coherencia, geométricamente corregidos, a formato raster para generar los productos cartográficos, tal y como se muestra en las Figuras 18 y 19.

Figura 18. Mapa de desplazamiento vertical (ascendente) de la ladera occidental del volcán Turrialba

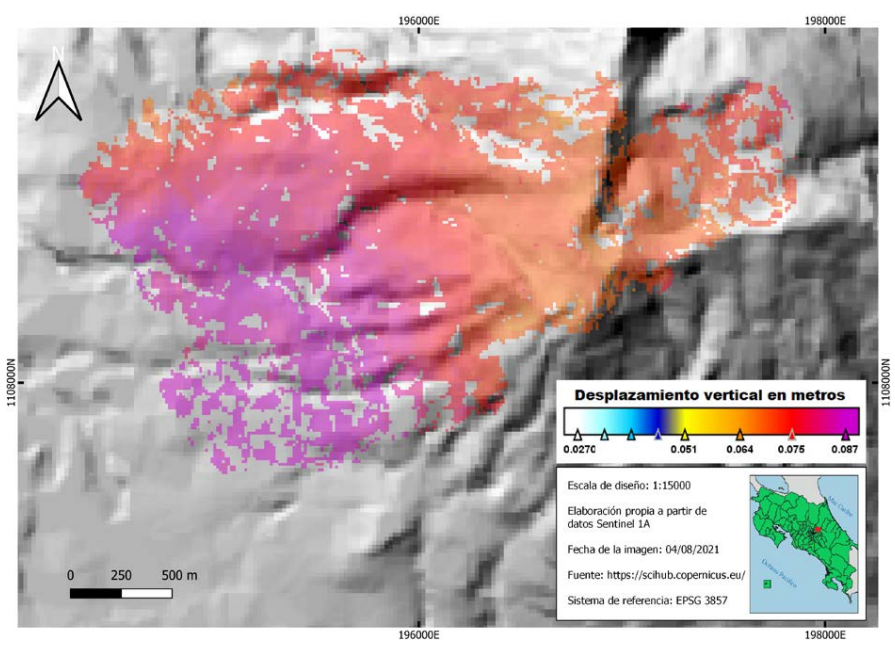

Figura 19. Mapa de coherencia interferométrica de la ladera occidental del volcán Turrialba

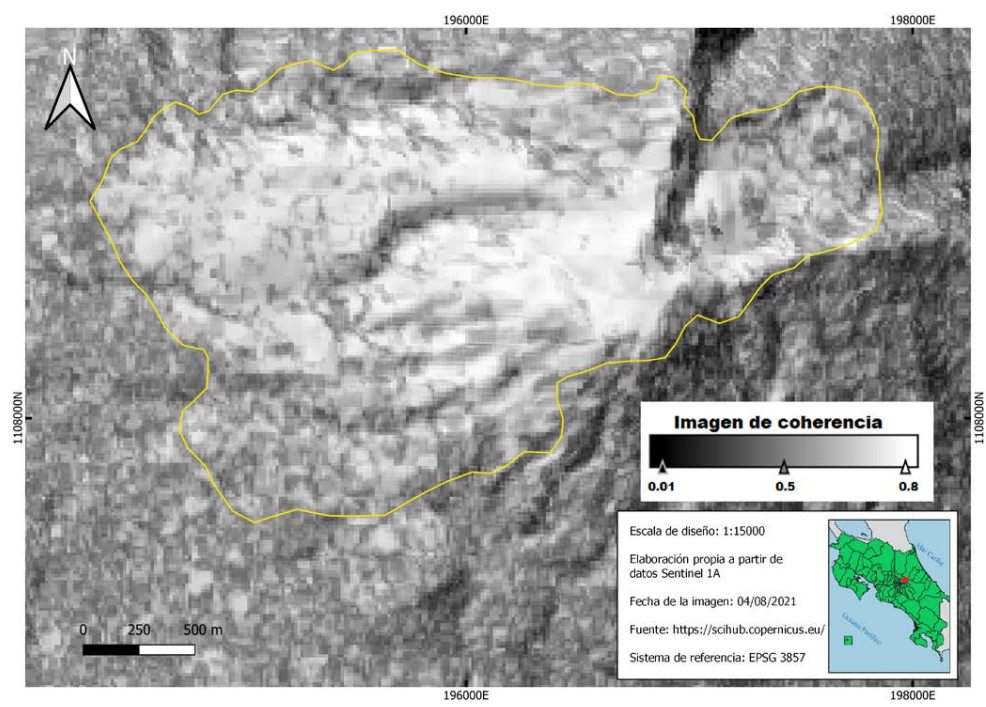




\section{Conclusiones}

La presente metodología revela que, a pesar de la general contracción y subsidencia del macizo Turrialba-Irazú (de acuerdo con el boletín semanal del Programa de Vigilancia Volcánica de 3 de agosto del 2020 del OVSICORI) tras un evento eruptivo como el acontecido el 1 de agosto de 2020 las faldas del cráter experimentaron una deformación ligeramente ascendente; de unos $7 \mathrm{~cm}$ en la cumbre (por encima de los $3090 \mathrm{~m}$ de altitud) hasta los $9 \mathrm{~cm}$ de las laderas del cráter (entre 2600 y $2900 \mathrm{~m}$ de altitud) tal y como indican las Figuras 18 y 19.

Como limitaciones cabe mencionar que no es posible atribuir exclusivamente a la erupción del 1 agosto las deformaciones estimadas. Se requeriría de un estudio más profundo para establecer correlaciones entre actividad sísmica, volcánica y deformación de la superficie. En todo caso, la robustez de los datos de deformación obtenidos viene respaldada por la imagen de coherencia (Figura 19) que indica valores superiores a 0,7. Cabe recordar en este sentido que la coherencia $(\gamma)$ es un parámetro cuya magnitud está relacionada con la calidad conseguida en la estimación de la fase interferométrica, y varía entre 0 (baja calidad) y 1 (alta calidad) (Herrera et al., 2009). Generalmente se obtienen altos valores de coherencia temporal en superficies con poca vegetación, tal y como sucede en la ladera occidental del volcán Turrialba, prácticamente desprovista de vegetación actualmente. Es importante, en todo caso, realizar las observaciones de imágenes SAR simultáneamente, o tan cercanas en el tiempo como sea posible (Siqueira, 2019) razón por la cual no se utilizaron otras imágenes Sentinel 1 en el presente estudio.

En cuanto a las condiciones meteorológicas, los cambios atmosféricos enmascaran u ocultan la parte de la fase interferométrica debida a los movimientos del terreno (Herrera et al., 2009). En este sentido el período transcurrido entre la adquisición de las dos imágenes se caracterizó por una canícula más o menos estable desde el 20 de julio hasta la primera semana de agosto (de acuerdo con el boletín de julio-setiembre de 2020 del Instituto Meteorológico de Costa Rica)

Finalmente, conviene recordar que las técnicas de interferometría diferencial con radar de apertura sintética se han desarrollado y validado en los últimos años en estudios de deslizamientos y movimientos sísmicos, 
pero su aplicación en la mejora de los modelos de predicción de esos eventos se encuentra aún en una fase incipiente.

\section{Referencias}

Amelung, F., Galloway, D.L., Bell, J.W., Zebker, H.A., \& Laczniak, R.J. (1999). Sensing the ups and downs of Las Vegas: InSAR reveals structural control of land subsidence and aquifer-system deformation. Geology, 27(6), 483-486.

Braun, A. \& Veci, L. (2015). Sentinel-1 Toolbox: TOPS Interferometry Tutorial. (2020) SkyWatch Space Applications Inc. European Space Agency. Braun, A. (2019). Radar satellite imagery for humanitarian response. Bridging the gap between technology and application. (Tesis doctoral). Mathematisch-Naturwissenschaftlichen Fakultät der Eberhard Karls Universität, (Tübingen). doi: http://doi. org/10.15496/publikation-32698

Cascini, L., Ferlisi, S., Fornaro, G., Lanari, R., Peduto, D. \&Zeni, G. (2006). Subsidence monitoring in Sarno urban area via multi-temporal DInSAR technique. Int. J. Remote Sens, 27(8), 1709-1716.

Chuvieco, E. (2010). Teledetección Ambiental. La observación de la Tierra desde el espacio ( $3^{\circ}$ ed.). Barcelona, España: Ariel Ciencia.

Elachi, C. (1987). Introduction to the Physics and Techniques of Remote Sensing. Chichester, New York, Brisbane, Toronto, Singapore: John Wiley. Geological Magazine, 125(4), 467-467. doi: http://doi. org/10.1017/S0016756800013133

Goldstein, R., Zebker, H., \& Werner, C. (1988). Satellite Radar Interferometry: Two-Dimensional Phase Unwrapping. Radio Science, 23(4), 713-720. doi: http://doi.org/10.1029/RS023i004p00713

González, G., Mora-Amador, R., Ramírez Umaña, C., Rouwet, D., Alpízar, Y., Picado, C., \& Mora, R. (2015). Actividad histórica y análisis de la amenaza del volcán Turrialba, Costa Rica. Revista Geológica de América Central, (52), 129-149. doi: http://doi.org/10.15517/ rgac.v0i52.19033

Guerrero, J. (2008). Dinámica fluvial y riesgos naturales derivados de la subsidencia kárstica en los valles del Huerva y del Ebro. (Tesis doctoral). Universidad de Zaragoza. 
Hanssen, R. (2001). Radar Interferometry Data Interpretation and Error Analysis. Kluwer Academic, Dordrecht, Boston. doi: http://doi. org/10.1007/0-306-47633-9

Instituto Meteorológico Nacional de Costa Rica. (2020). Boletín Meteorológico Mensual, agosto 2020.

Herrera, G., Tomás, R., Lopez-Sanchez, J., Monserrat, O., Cooksley, G. \& Mulas, J. (2009). Sistemas radar aplicados a la investigación de subsidencia y movimientos de ladera. Enseñanza de las Ciencias de la Tierra, (17.3), 316-324.

Herrera, G., Tomas, R., López-Sánchez, J.M., Delgado, J., Mallorquí, J.J., Duque, S., \& Mulas, J. (2007). Advanced DInSAR analysis on mining areas: La Unión case study (Murcia, SE Spain). Eng. Geol. 90(3-4), 148-159.

Jiménez Perálvarez, J. D. (2013). Movimientos de ladera en la vertiente meridional de Sierra Nevada (Granada, España): identificación, análisis y cartografia de susceptibilidad y peligrosidad mediante SIG. (Tesis doctoral). Universidad de Granada, España. Recuperado de: http://hdl.handle.net/10481/23770

Klees, R. \& Massonnet, D. (1999). Deformation measurements using SAR interferometry: potential and limitations. Geologie en Mijnbouw 77, 161-176.

Massonnet, D. \& Feigl, M. (1998). Radar interferometry and its application to changes in the earth's surface. Review of Geophysics, 36(4): 441-500.

Massonnet, D., Rossi, M., Carmona, C., Adragna, F., Peltzer, G., Feigl, K.\& Rabaute, T. (1993). The displacement field of the Landers earthquake mapped by radar interferometry. Nature, 364: 138-142.

Moreira, A., Prats-Iraola, P., Younis, M., Krieger, G., Hajnsek, I. \&Papathanassiou, K. (2013). A Tutorial on Synthetic Aperture Radar. IEEE Geoscience and Remote Sensing Magazine (GRSM), 1(1), 6-43. doi: http://doi.org/10.1109/MGRS.2013.2248301

Observatorio Vulcanológico y Sismológico de Costa Rica. (2020). Boletín semanal del Programa de Vigilancia Volcánica del 3 de agosto del 2020. Universidad Nacional de Costa Rica.

Richards M. A., Scheer J. A. \& Holm, W. A. (2010). Principles of Modern Radar: Basic Principles. Unites States: SciTech Publishing. 
Sigmudsson, F., Durand, P. \& Massonnet, D. (1999). Opening of an eruptive fissure and seaward displacement at Piton de la Fournaise volcano measured by Radarsat satellite radar interferometry. Geophys. Res. Lett., 26(5), 533-536.

Siqueira, P. (2019). Forest Stand Height Estimation. SAR Handbook: Comprehensive Methodologies for Forest Monitoring and Biomass Estimation. A. Flores, K. Herndon, R. Thapa, E. Cherrington. (Eds.). E. NASA. doi: http://doi.org/10.25966/4530-7686 
